1966-12-01

\title{
Equation of State of Sodium Chloride
}

\author{
Daniel L. Decker \\ dldecker@broadweave.net
}

Follow this and additional works at: https://scholarsarchive.byu.edu/facpub

Part of the Astrophysics and Astronomy Commons, and the Physics Commons

\section{Original Publication Citation}

Decker, D. L. "Equation of State of Sodium Chloride." Journal of Applied Physics 37 (1966):

512-514.

\section{BYU ScholarsArchive Citation}

Decker, Daniel L., "Equation of State of Sodium Chloride" (1966). Faculty Publications. 805.

https://scholarsarchive.byu.edu/facpub/805 


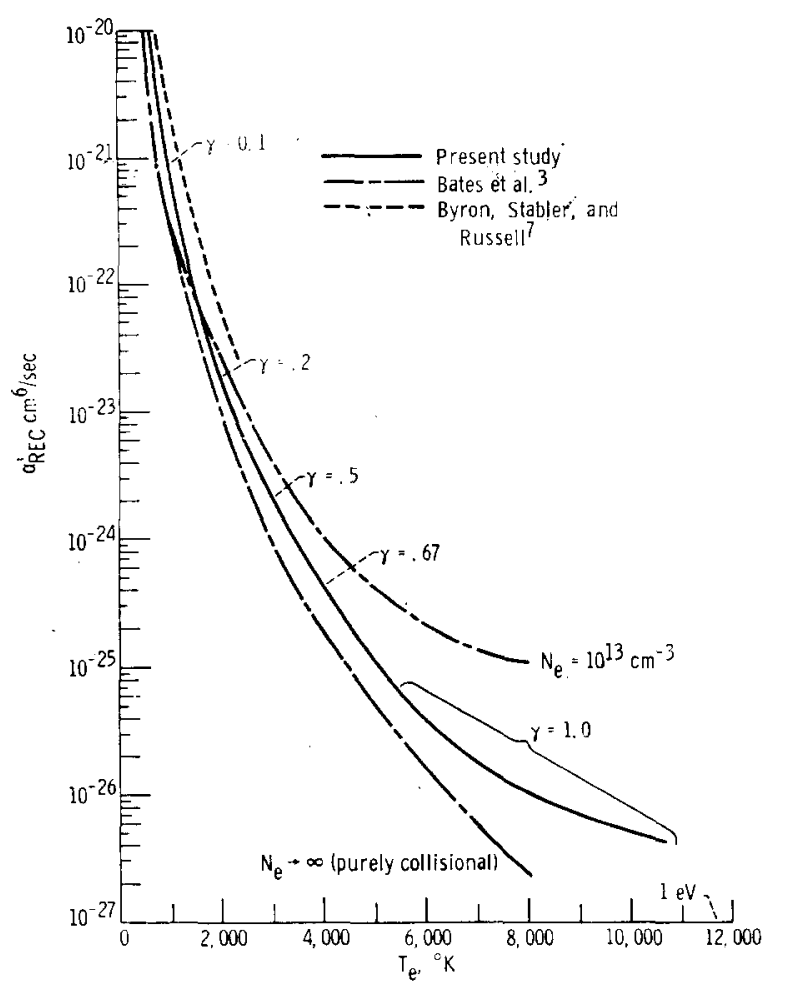

FIG. 3. Argon and hydrogen three-body collisional recombination coefficients as functions of electron temperature for range of electron number density $\left(10^{\left.13-10^{18} \mathrm{~cm}^{-3}\right)}\right.$

The three-body coefficient $\alpha_{\text {rec }}$ from several sources is plotted versus $T_{e}$ in Fig. 1 . For the curve from Ref. 8, the quantum gaps which correspond to the minimum de-excitation rates are indicated. The dashed curve gives the results of Ref. 7 for potassium, while the broken curves from Ref. 3 are for a pseudoalkali atom plasma. The coefficient from Ref. 3 is a function of number density $N_{e}$ because radiative de-excitation and two-body capture are included. The experimental results of $\mathrm{Cool}$ and Zukoski ${ }^{9}$ for potassium-seeded argon with $T_{e}$ from $1800^{\circ}$ to $3200^{\circ} \mathrm{K}$ agree closely with the results of Ref. 8. Experimental points are shown for both pure cesium and cesium-seeded argon. ${ }^{10-12}$ The agreement between theory and experiment for the alkalies is well within the expected accuracy of the Gryzinski cross sections. ${ }^{3,8}$

The disparity between the theoretical ${ }^{13}$ (Gryzinski, total excitation) and experimental ${ }^{14}$ (first excitation, $\simeq 90 \%$ total excitation) monoenergetic cross sections (in $\mathrm{cm}^{2}$ ) and Maxwell averaged total excitation coefficients (in $\mathrm{cm}^{3} / \mathrm{sec}$ ) can be seen for potassium and cesium from Fig. 2. The agreement will be less satisfactory for nonhydrogenic atoms and there may be some ambiguity as to choice of upper states for excitation calculations. Using more accurate excitation cross sections may not always improve the recombination calculation because radiative emission may be important in both recombination experiments and MHD systems. The contribution of this deexcitation mechanism depends on the superelastic collision rate and optical absorption cross section of the ionized species. ${ }^{2,7,8}$

Values of $\alpha_{\text {rec }}$ for argon were calculated in the same fashion as the cesium coefficients. At the lower electron temperatures, $500^{\circ}$ to $3000^{\circ} \mathrm{K}$, the $\gamma$ factor was fixed by fitting the results between the curves from Refs. 3 and 4 . This procedure is necessary because of the approximation of "collapsed" quantum states.

The $\alpha_{\text {rec }}$ ' for argon is plotted vs $T_{0}$ in Fig. 3 and compared with the hydrogen results of Refs. 3 and 7 . Some discrepancy between the results of Ref. 3 and the present study is due to the difference between the Maxwell averaged first excitation cross sections of argon and hydrogen. The neglect of radiative deexcitation in the present calculation is of less importance, as was pointed out in Ref. 8 where the mean radiative transition probabilities were compared with the superelastic collision frequencies. ${ }^{8}$ It has been shown that the purely collisional approach is a good approximation for cesium, ${ }^{8}$ while the maximum radiative correction for argon is $50 \%$ at $T_{t}=10000^{\circ} \mathrm{K}$.

For nonequilibrium MHD power generation, recombination rates will not be very different for carrier and seed gases at achievable electron temperatures. ${ }^{1}$ However, the average energy lost by the free electrons in recombination will depend on the mechanism by which the captured electrons become deexcited. Results of Ref. 2 indicate that emission of radiation is not an important energy loss process for potassium in argon. Collisional deexcitation frequencies from Ref. 8 indicate that this is also true for cesium in argon. However, a more complete study of the plasma energy balance incorporating accurate cross sections for ionization, excitation, and resonance absorption is desirable.

1 J. E. Heighway, and L. D. Nichols, NASA TN D-2651 (February 1965) 2 T. Hiramoto, J. Phys. Soc. Japan 20, 1061 (1965)

${ }^{3}$ D. R. Bates, A. E. Kingston, and R. W. P. McWhirter, Proc. Roy. Soc (London) A-267, 297 (1962).

4S. Byron, R. C. Stabler, and P. I. Bortz, Phys. Rev. Letters 8, 376

5 R. H. Fowler, Statistical Mechanics (Cambridge University Press, New York, 1936), 2nd, ed., Chap. 17.

M. Gryzinski. Phys. Rev. 138, A305 (1965)

7 S. Byron, P. Bortz, and G. Russell, Proc. of 4th Symp. on Eng. Aspects of MHD, Univ. Calif. (10-11 April 1963).

J. V. Dugan, Jr., NASA TN D-2004 (October 1964)

${ }^{9} \mathrm{~T}$. A. Cool and E. E. Zukoski, Proc. of 6th Symp. on Eng. Aspects of 10 , Univ. of Pitts. (21-22 April 1965)

11 J. Y. Wada and R. C. Knechtli, Phys. Rev. Letters 10, 513 (1963). ${ }_{12} \mathrm{Yu}$. M. Aleskovskii, Soviet Phys. -JETP 17, $570(1963)$. ${ }_{13}$ L. P. Harris, Rep. 64-RL-3698G, Gen. Elec. Co. (June 1964) $13 \mathrm{~J}$. W. Sheldon and J. V. Dugan, Jr.. J. Appl. Phys. 36, 650 (1965). 14 I. P. Zapesochny and L. L. Shimon: Abstracts of $1 V$ th Internat'l Conf City, Canada (2-6 August 1965), p. 401.

\section{Equation of State of Sodium Chloride}

\section{L. DECKER}

Department of Physics, Brigham Young University, Provo, Ulah (Received 8 August 1966)

$B^{\mathrm{E}}$ ECAUSE of many requests, I wish to publish the following numerical table of results for the equation of state of $\mathrm{NaCl}$ as calculated in an earlier paper ${ }^{1}$ along with a few comments on various proposed equations for $\mathrm{NaCl}$. Table I gives the pressure in kilobars at the corresponding values of $\Delta a / a_{0}$ and temperature in the appropriate row and column. The parameter $\Delta a / a_{0}$ is the fractional compression of the lattice parameter where the standard value $a_{0}$ is the appropriate lattice parameter at zero pressure and $25^{\circ} \mathrm{C}$. The increments between the values given in the table were chosen such that one can linearly interpolate between the tabulated values to an accuracy of better than $0.02 \mathrm{kbar}$.

In recent years several equations have been proposed to give the volume change vs pressure for $\mathrm{NaCl}$ at room temperature., A comparison of these equations and the results in Table I is given in Fig. 1 along with experimental measurements by Bridgman, ${ }^{4}$ Christian, ${ }^{5}$ and Perez-Albuerne and Drickamer. ${ }^{3}$ This figure gives the differences in $\Delta V / V_{0}$ of the respective equations or experimental measurements at a specified pressure to the $\Delta V / V_{0}$ calculated in Ref. 1. at that pressure. Experimental values of $\mathrm{NaCl}$ compression have been measured by Jeffery $e t a l .^{6}$ at the pressure of the bismuth I-II phase transition. If the value of $25.4 \mathrm{kbar}^{7}$ is accepted for the Bi I-II transition, then Jeffery's measurement of $\Delta V / V_{0}=-0.084 \pm 0.02$ for the compression of $\mathrm{NaCl}$ at this transition gives one point at which both $P$ and $V$ are simultaneously known. This point is shown by the large square in Fig. 1. It is noted that all results agree to within the accuracy of the x-ray measurement at 25.4 kbar except Murnaghan's equation em- 
TABLE I. Calculated pressure at given compressions and temperatures from $0-500 \mathrm{kbar}$ and $0^{\circ} \mathrm{C}-\mathrm{mp}$ for $\mathrm{NaCl}$. Based on equation of state by Decker.

\begin{tabular}{|c|c|c|c|c|c|c|c|c|c|c|c|c|c|c|c|c|c|}
\hline$\frac{\Delta a}{\Delta_{0}}$ & $0 \mathrm{C}$ & $25 \mathrm{C}$ & $100 \mathrm{C}$ & $200 \mathrm{C}$ & $300 \mathrm{C}$ & $400 \mathrm{C}$ & $500 \mathrm{C}$ & $600 \mathrm{C}$ & $700 \mathrm{C}$ & $800 \mathrm{C}$ & $900 \mathrm{C}$ & $1000 \mathrm{C}$ & $1200 \mathrm{C}$ & $1400 \mathrm{C}$ & $1600 \mathrm{C}$ & $1800 \mathrm{C}$ & $2000 \mathrm{C}$ \\
\hline 0.040 & & & & & & & & & & 0.45 & & & & & & & \\
\hline 0.038 & & & & & & & & & & 1.25 & 4.03 & & & & & & \\
\hline $\begin{array}{l}0.036 \\
0.034\end{array}$ & & & & & & & & & & 2.09 & 4.87 & & & & & & \\
\hline $\begin{array}{l}0.034 \\
0.032\end{array}$ & & & & & & & & & $\begin{array}{l}0.15 \\
1.03\end{array}$ & $\begin{array}{l}2.94 \\
3.83\end{array}$ & $\begin{array}{l}5.74 \\
6.63\end{array}$ & & & & & & \\
\hline $\begin{array}{l}0.030 \\
0.028\end{array}$ & & & & & & & & & 1.94 & 4.75 & 7.55 & 10.36 & & & & & \\
\hline $\begin{array}{l}0.028 \\
0.026\end{array}$ & & & & & & & & $\begin{array}{l}0.07 \\
1.03\end{array}$ & 2.88 & $\begin{array}{l}5.69 \\
6.67\end{array}$ & $\begin{array}{l}8.51 \\
9.49\end{array}$ & $\begin{array}{l}11.32 \\
12.31\end{array}$ & & & & & \\
\hline $\begin{array}{l}0.020 \\
0.024 \\
0.022\end{array}$ & & & & & & & & $\begin{array}{l}1.05 \\
2.02\end{array}$ & $\begin{array}{l}3.85 \\
4.83\end{array}$ & $\begin{array}{l}0.01 \\
7.67\end{array}$ & $\begin{array}{r}9.49 \\
10.50\end{array}$ & 13.33 & & & & & \\
\hline $\begin{array}{l}0.022 \\
0.020\end{array}$ & & & & & & & 0.21 & 3.04 & 5.87 & 8.71 & 11.55 & 14.39 & & & & & \\
\hline $\begin{array}{l}0.020 \\
0.018\end{array}$ & & & & & & & 1.25 & 4.10 & 6.93 & 9.78 & 32.62 & 15.47 & & & & & \\
\hline $\begin{array}{l}0.018 \\
0.016\end{array}$ & & & & & & 0.60 & $\begin{array}{r}2.33 \\
3.45\end{array}$ & 5.18 & 8.03 & $\begin{array}{l}10.88 \\
12.02\end{array}$ & $\begin{array}{r}13.74 \\
14.88\end{array}$ & 16.59 & 23.47 & & & & \\
\hline 0.014 & & & & & & $\begin{array}{l}1.74 \\
1.74\end{array}$ & $\begin{array}{l}3.45 \\
4.56\end{array}$ & $\begin{array}{l}6.30 \\
7.46\end{array}$ & $\begin{array}{r}9.16 \\
10.32\end{array}$ & $\begin{array}{l}12.02 \\
13.29\end{array}$ & $\begin{array}{l}14.00 \\
26.06\end{array}$ & $\begin{array}{l}17.74 \\
18.93\end{array}$ & 24.67 & & & & \\
\hline 0.012 & & & & & 0.06 & 2.92 & 5.78 & 8.65 & 11.52 & 14.40 & 17.28 & 20.15 & 25.91 & & & & \\
\hline $\begin{array}{l}0.010 \\
0.008\end{array}$ & & & & & 1.26 & 4.13 & 7.00 & 9.88 & 12.76 & 15.64 & 18.53 & 21.41 & 27.19 & & & & \\
\hline $\begin{array}{l}0.008 \\
0.006\end{array}$ & & & & & 2.50 & 5.38 & 8.26 & 11.15 & 14.03 & 16.93 & 19.82 & 22.71 & 28.50 & & & & \\
\hline $\begin{array}{l}0.006 \\
0.004\end{array}$ & & & & 0.92 & 3.79 & 6.67 & 9.56 & 12.45 & 15.35 & 18.25 & 21.15 & 24.05 & 29.86 & & & & \\
\hline $\begin{array}{l}0.004 \\
0.002\end{array}$ & & & & 2.24 & 5.11 & $8 . \infty 0$ & 10.90 & 13.80 & 16.70 & 19.61 & 22.52 & 25.43 & 31.25 & & & & \\
\hline $\begin{array}{l}0.002 \\
0.000\end{array}$ & & & 0.73 & 3.59 & 6.47 & 9.37 & 12.27 & 15.18 & 18.09 & 21.01 & 23.92 & 26.84 & 32.68 & $400 ?$ & & & \\
\hline $\begin{array}{l}-0.002 \\
-0.004\end{array}$ & 0.74 & $\begin{array}{l}0.00 \\
1.43\end{array}$ & $\begin{array}{l}2.12 \\
3.56\end{array}$ & $\begin{array}{l}4.99 \\
6.43\end{array}$ & $\begin{array}{l}7.88 \\
9.33\end{array}$ & $\begin{array}{l}10.78 \\
12.24\end{array}$ & $\begin{array}{l}13.69 \\
15.16\end{array}$ & $\begin{array}{l}16.01 \\
18.08\end{array}$ & $\begin{array}{l}19.53 \\
21.01\end{array}$ & $\begin{array}{l}22.45 \\
23.94\end{array}$ & $\begin{array}{l}25.38 \\
26.87\end{array}$ & $\begin{array}{l}28.03 \\
29.80\end{array}$ & $\begin{array}{l}34.16 \\
35.68\end{array}$ & $\begin{array}{l}40.02 \\
41.56\end{array}$ & & & \\
\hline $\begin{array}{l}-0.004 \\
-0.006\end{array}$ & 2.21 & 2.91 & $\begin{array}{l}3.50 \\
5.03\end{array}$ & $\begin{array}{l}6.43 \\
7.91\end{array}$ & $\begin{array}{r}9.35 \\
20.82\end{array}$ & $\begin{array}{l}12.24 \\
13.74\end{array}$ & $\begin{array}{l}17.10 \\
16.66\end{array}$ & $\begin{array}{l}10.00 \\
19.59\end{array}$ & 22.53 & $\begin{array}{l}23.94 \\
25.47\end{array}$ & $\begin{array}{l}20.01 \\
28.41\end{array}$ & 31.34 & 37.24 & 43.13 & & & \\
\hline $\begin{array}{l}-0.006 \\
-0.008\end{array}$ & $\begin{array}{l}3.72 \\
5.28\end{array}$ & 4.42 & 6.56 & 9.44 & 12.35 & 15.28 & 18.21 & 21.15 & 24.10 & 27.04 & 29.99 & 32.94 & 38.84 & 44.75 & & & \\
\hline $\begin{array}{l}-0.008 \\
-0.010 \\
-0.012\end{array}$ & $\begin{array}{l}5.28 \\
6.89\end{array}$ & $\begin{array}{l}5.99 \\
7.60\end{array}$ & $\begin{array}{l}8.12 \\
9.74\end{array}$ & $\begin{array}{l}11.02 \\
12.64\end{array}$ & $\begin{array}{l}23.93 \\
25.56\end{array}$ & $\begin{array}{l}16.87 \\
18.50\end{array}$ & 19.81 & 22.76 & 25.71 & 28.66 & 31.62 & 34.58 & 40.50 & 46.42 & & & \\
\hline $\begin{array}{l}-0.010 \\
-0.012 \\
-0.014\end{array}$ & $\begin{array}{l}6.09 \\
8.55\end{array}$ & $\begin{array}{l}7.60 \\
9.26\end{array}$ & $\begin{array}{r}9.74 \\
11.40\end{array}$ & $\begin{array}{l}12.64 \\
14.38\end{array}$ & $\begin{array}{l}25.56 \\
27.24\end{array}$ & $\begin{array}{l}18.50 \\
20.19\end{array}$ & $\begin{array}{l}21.45 \\
23.15\end{array}$ & $\begin{array}{l}24 . \\
26 .\end{array}$ & $\begin{array}{l}27.37 \\
29.01\end{array}$ & 30.33 & 33.30 & 36.26 & 42.20 & 48.14 & & & \\
\hline $\begin{array}{l}-0.014 \\
-0.016\end{array}$ & 10.26 & 10.97 & 13.12 & $\begin{array}{l}14.30 \\
16.03\end{array}$ & 18.97 & 21.92 & $\begin{array}{l}23.15 \\
24.89\end{array}$ & $\begin{array}{l}26.11 \\
27.86\end{array}$ & $\begin{array}{l}29.01 \\
30.84\end{array}$ & $\begin{array}{l}32.05 \\
33.81\end{array}$ & $\begin{array}{l}35.02 \\
36.80\end{array}$ & $\begin{array}{l}37.99 \\
39.78\end{array}$ & $\begin{array}{l}43.95 \\
45.75\end{array}$ & $\begin{array}{l}49.90 \\
51.72\end{array}$ & & & \\
\hline $\begin{array}{l}-0.016 \\
-0.018\end{array}$ & 12.02 & 12.73 & 14.88 & 17.80 & 20.75 & 23.71 & 26.68 & 29.66 & 32.65 & 35.63 & 38.62 & $\begin{array}{l}39.10 \\
41.62\end{array}$ & 47.60 & 53.59 & & & \\
\hline $\begin{array}{l}-0.018 \\
-0.020\end{array}$ & 13.83 & 14.54 & 16.70 & 19.62 & 22.58 & 25.55 & 28.53 . & 31.51 & 34.51 & 37.50 & 40.50 & 43.50 & 49.50 & 55.51 & 61.52 & & \\
\hline & 25.69 & 16.40 & 18.56 & 21.50 & 24.46 & 27.44 & $30.43^{\circ}$ & 33.42 & 36.42 & 39.43 & 42.39 & 45.45 & 51.46 & 57.48 & 63.51 & & \\
\hline $\begin{array}{l}-0.022 \\
-0.024\end{array}$ & $\begin{array}{l}17.61 \\
19.59\end{array}$ & $\begin{array}{l}18.32 \\
20.30\end{array}$ & 20.49 & $\begin{array}{l}23.43 \\
25.42\end{array}$ & $\begin{array}{l}26.40 \\
28.40\end{array}$ & $\begin{array}{l}29.39 \\
31.39\end{array}$ & 32.38 & 35.38 & $\begin{array}{l}38.39 \\
40.42\end{array}$ & $\begin{array}{l}41.40 \\
43.44\end{array}$ & $\begin{array}{l}44.43 \\
46.46\end{array}$ & $\begin{array}{l}47.44 \\
49.49\end{array}$ & $\begin{array}{l}53.48 \\
55.54\end{array}$ & & 65.55 & & \\
\hline $\begin{array}{l}-0.026 \\
-0.028\end{array}$ & 21.62 & $\begin{array}{l}20.30 \\
22.33\end{array}$ & $\begin{array}{l}22.47 \\
24.51\end{array}$ & $\begin{array}{l}25.42 \\
27.46\end{array}$ & $\begin{array}{l}28.40 \\
30.45\end{array}$ & $\begin{array}{l}31.39 \\
33.45\end{array}$ & $\begin{array}{l}34.39 \\
36.46\end{array}$ & $\begin{array}{l}37.40 \\
39.48\end{array}$ & $\begin{array}{l}40.42 \\
42.50\end{array}$ & $\begin{array}{l}43.44 \\
45.53\end{array}$ & $\begin{array}{l}40.40 \\
48.57\end{array}$ & $\begin{array}{l}49.49 \\
51.60\end{array}$ & 57.67 & $\begin{array}{l}61.59 \\
63.74\end{array}$ & $\begin{array}{l}67.65 \\
69.81\end{array}$ & & \\
\hline $\begin{array}{l}-0.028 \\
-0.030\end{array}$ & 23.71 & 24.43 & 26.61 & 29.57 & 32.56 & 35.57 & 38.59 & 41.62 & 44.65 & 47.68 & 50.71 & 53.77 & 59.86 & 65.94 & 72.03 & & \\
\hline $\begin{array}{l}-0.030 \\
-0.032\end{array}$ & $\begin{array}{l}25.87 \\
28.08\end{array}$ & $\begin{array}{l}26.58 \\
28.80\end{array}$ & 28.77 & 31.73 & 34.73 & 37.75 & 40.78 & 43.81 & 46.85 & 49.90 & 52.95 & 55.00 & 62.10 & 68.20 & 74.31 & & \\
\hline $\begin{array}{l}-0.034 \\
-0.036\end{array}$ & $\begin{array}{l}28.08 \\
30.36\end{array}$ & $\begin{array}{l}28.80 \\
31.08\end{array}$ & $\begin{array}{l}30.00 \\
33.27\end{array}$ & $\begin{array}{l}33.66 \\
36.25\end{array}$ & 36.97 & $\begin{array}{l}39.99 \\
42.30\end{array}$ & $\begin{array}{l}43.03 \\
45.34\end{array}$ & $\begin{array}{l}46.07 \\
48.39\end{array}$ & $\begin{array}{r}49.12 \\
51.45\end{array}$ & 52.17 & $\begin{array}{l}55.24 \\
57.48\end{array}$ & $\begin{array}{l}58.29 \\
60.66\end{array}$ & $\begin{array}{l}64.41 \\
66.78\end{array}$ & $\begin{array}{l}70.53 \\
72.92\end{array}$ & $\begin{array}{l}76.65 \\
79.06\end{array}$ & 85.20 & \\
\hline $\begin{array}{l}-0.036 \\
-0.038\end{array}$ & 32.71 & $\begin{array}{l}53.0 \\
33.4\end{array}$ & $\begin{array}{l}33.27 \\
35.62\end{array}$ & $\begin{array}{l}36.25 \\
38.61\end{array}$ & $\begin{array}{l}39.26 \\
41.63\end{array}$ & $\begin{array}{l}42.30 \\
44.67\end{array}$ & $\begin{array}{l}45.34 \\
47.72\end{array}$ & $\begin{array}{l}48.39 \\
50.78\end{array}$ & $\begin{array}{l}51.45 \\
53.85\end{array}$ & $\begin{array}{l}54.51 \\
56.91\end{array}$ & $\begin{array}{l}57.48 \\
60.00\end{array}$ & $\begin{array}{l}10.06 \\
63.08\end{array}$ & $\begin{array}{l}60.10 \\
69.22\end{array}$ & $\begin{array}{l}72.92 \\
75.37\end{array}$ & 81.53 & 87.69 & \\
\hline $\begin{array}{l}-0.038 \\
-0.040 \\
-0.042\end{array}$ & $\begin{array}{l}35.12 \\
37.60\end{array}$ & $\begin{array}{l}35.84 \\
38.32\end{array}$ & 38.04 & 41.03 & 44.06 & 47.11 & 50.27 & 53.23 & 56.31 & 59.39 & 62.48 & 65.56 & 71.74 & 77.89 & 84.07 & 90.25 & \\
\hline $\begin{array}{l}-0.040 \\
-0.042 \\
-0.044\end{array}$ & $\begin{array}{r}37.60 \\
40.15\end{array}$ & $\begin{array}{l}38.32 \\
40.87\end{array}$ & $\begin{array}{l}40.53 \\
43.08\end{array}$ & $\begin{array}{l}43.52 \\
46.09\end{array}$ & $\begin{array}{l}46.56 \\
49.13\end{array}$ & $\begin{array}{l}49.61 \\
52.19\end{array}$ & $\begin{array}{l}52.68 \\
55.27\end{array}$ & $\begin{array}{l}55.76 \\
58.35\end{array}$ & $\begin{array}{l}58.84 \\
61.44\end{array}$ & $\begin{array}{l}61.93 \\
64.54\end{array}$ & $\begin{array}{l}64.99 \\
67.65\end{array}$ & $\begin{array}{l}68.11 \\
70.74\end{array}$ & $\begin{array}{l}74.30 \\
76.95\end{array}$ & $\begin{array}{l}80.48 \\
83.15\end{array}$ & $\begin{array}{l}86.68 \\
89,36\end{array}$ & 92.88 & \\
\hline $\begin{array}{l}-0.044 \\
-0.046\end{array}$ & 42.78 & 43.50 & 45.71 & $\begin{array}{l}46.09 \\
48.72\end{array}$ & 51. & $\begin{array}{l}52.19 \\
54.84\end{array}$ & $\begin{array}{l}53 . \\
57 .\end{array}$ & $\begin{array}{l}58.35 \\
61.02\end{array}$ & 61.44 & $\begin{array}{l}64.54 \\
67.22\end{array}$ & $\begin{array}{l}67.65 \\
70.33\end{array}$ & $\begin{array}{l}70.74 \\
72.67\end{array}$ & $\begin{array}{l}76.95 \\
79.51\end{array}$ & $\begin{array}{l}83.15 \\
85.89\end{array}$ & $\begin{array}{l}89.36 \\
92.11\end{array}$ & $\begin{array}{l}95.57 \\
98.34\end{array}$ & $\begin{array}{l}101.79 \\
104.58\end{array}$ \\
\hline $\begin{array}{l}-0.046 \\
-0.048\end{array}$ & 45.47 & 46.20 & 48.41 & 51.43 & 54.49 & 57.57 & 60. & 63.76 & 66.86 & 69.96 & 73.10 & 76.21 & 82.45 & 88.69 & 94.94 & 101.19 & 107.44 \\
\hline $\begin{array}{l}-0.048 \\
-0.050\end{array}$ & 48.25 & 48.97 & 51.19 & 54. & 57.28 & 60.3 & 63. & 66.57 & 69.69 & 72.81 & 75.94 & 79.08 & 85. & 91 & 07 & 104.11 & 110.37 \\
\hline $\begin{array}{l}-0.050 \\
-0.052\end{array}$ & 51.10 & 51.82 & 54.05 & 57.08 & 60.15 & 63. & 66. & 69.47 & 72.59 & 75.72 & 78.86 & 82.01 & 88.26 & 94 & 100.82 & 107.10 & 113.39 \\
\hline $\begin{array}{l}0.052 \\
0.054\end{array}$ & 54.03 & $54.7^{8}$ & 56.98 & 60.02 & 63.10 & 66. & 69. & 72.44 & 75.57 & 78.71 & 81.86 & 85.00 & 91.29 & 97 & 103.88 & 110.18 & $\begin{array}{l}116.49 \\
119.66\end{array}$ \\
\hline $\begin{array}{l}-0.054 \\
-0.056\end{array}$ & 57.04 & 57.80 & 60.00 & 63.05 & 66.13 & 69.2 & 72.36 & 75.50 & 78.64 & 81.78 & 84.94 & 88.09 & 94.35 & 100 & 107.02 & $\begin{array}{l}113.34 \\
116.59\end{array}$ & 119.66 \\
\hline $\begin{array}{l}-0.056 \\
-0.058\end{array}$ & $\begin{array}{l}60.14 \\
63.32\end{array}$ & $\begin{array}{l}60.87 \\
64.05\end{array}$ & $\begin{array}{l}63.10 \\
66.29\end{array}$ & $\begin{array}{l}66.15 \\
69.35\end{array}$ & $\begin{array}{l}69.25 \\
72.45\end{array}$ & $\begin{array}{l}72.36 \\
75.57\end{array}$ & $\begin{array}{l}75.50 \\
78.71\end{array}$ & $\begin{array}{l}78.64 \\
81.86\end{array}$ & $\begin{array}{l}81.79 \\
85.02\end{array}$ & $\begin{array}{l}.84 .94 \\
88.18\end{array}$ & $\begin{array}{l}88.11 \\
91.36\end{array}$ & $\begin{array}{l}91.27 \\
94.54\end{array}$ & $\begin{array}{r}97.59 \\
100.87\end{array}$ & $\begin{array}{l}103.91 \\
107.27\end{array}$ & 113.56 & $\begin{array}{l}116.59 \\
119.92\end{array}$ & $\begin{array}{l}122.92 \\
126.27\end{array}$ \\
\hline $\begin{array}{l}-0.060 \\
-0.062\end{array}$ & $\begin{array}{l}63.32 \\
66.59\end{array}$ & $\begin{array}{l}64.05 \\
67.32\end{array}$ & $\begin{array}{l}60.29 \\
69.57\end{array}$ & $\begin{array}{l}09.35 \\
72.63\end{array}$ & $\begin{array}{l}1<.47 \\
75.7^{4}\end{array}$ & 78.87 & 82.02 & 85.17 & 88.34 & 91.51 & 94.70 & 97.88 & 104.24 & & & 123.33 & 129.71 \\
\hline $\begin{array}{l}-0.062 \\
-0.064\end{array}$ & 69.95 & 70.68 & 72.93 & 76.00 & 79.1 & 82.2 & 85.41 & 38.58 & 92.75 & 94.91 & 98.13 & 101.31 & 107.69 & & 12 & 126 & 133.24 \\
\hline $\begin{array}{l}-0.064 \\
-0.0666\end{array}$ & 73.41 & 74.14 & 76.39 & 79. & 82.5 & 85. & 88. & 92. & 95 & 98.44 & 101.66 & & 11 & 11 & 04 & 130 & 136.86 \\
\hline $\begin{array}{l}-0.066 \\
-0.068\end{array}$ & 76.96 & 77.69 & 79.94 & 83. & 85.1 & 89. & 92.4 & 95.67 & 0 & 102.85 & 105.2 & 10 & 114 & & 12 & $13^{h}$ & 140.57 \\
\hline $\begin{array}{l}-0.068 \\
-0.070\end{array}$ & 80.60 & 81. & 83. & 86. & 89. & 92. & 96.16 & 99.35 & 102.55 & 105.76 & 108.98 & 122.19 & & & & 13 & 144.38 \\
\hline $\begin{array}{l}-0.070 \\
-0.072\end{array}$ & 8 & 85. & 87. & 90.4 & 93. & $a t_{1}$ & 99. & 103.14 & & 109.56 & 112.79 & 11 & 12 & 12 & & 141 & 148.30 \\
\hline $\begin{array}{l}-0.072 \\
-0.074\end{array}$ & 88.20 & 88.9 & 91.20 & 94. & 97. & & 103. & 107.03 & 110.25 & 113.47 & 116 & 115 & 12 & & & 145.83 & 152.31 \\
\hline $\begin{array}{l}-0.074 \\
-0.076\end{array}$ & 92.15 & 28 & 95.15 & 98.26 & 101.43 & 104. & 107.81 & 111.03 & & 117.48 & 120.73 & 12 & & & & 145 & 156.43 \\
\hline-0.076 & 0631 & 96.95 & 99.22 & 102.33 & 105. & 108 & 111.90 & 15.23 & & 121.60 & 124 & & & 14. & & & 160.66 \\
\hline-0.078 & 100.3 & 101.12 & 103.39 & 106.51 & 109. & 112 & 116.11 & 119. & & 125 & 08 & & 138 & & 15 & & 165.00 \\
\hline-0.080 & 104.66 & 105.40 & 107.68 & 110.80 & & & & 123 & 126 & 130 & & & & & & & 169.45 \\
\hline-0.082 & 109.06 & 109.9 & 112.08 & 115. & 118.40 & 121 & 124.86 & 128.11 & 131.37 & 134.63 & 137.90 & 19 & 14 & & & & 174.02 \\
\hline-0.084 & 113.58 & 1.14 .32 & 316.60 & 119. & 122. & 126 & 129.41 & & & & 242 & .79 & & & 16 & & 178.70 \\
\hline-0.086 & 118.2 & 118.96 & 121.24 & 324. & 127. & 130 & & & & & 247 & & & & & & 183.51 \\
\hline-0.088 & 122.98 & 123.72 & 126.01 & 129.16 & 132.37 & 135. & 138.89 & & 145.44 & 148.73 & 152.04 & 15 & 161 & & 175 & & 188.44 \\
\hline-0.090 & 127.87 & 128.61 & 130.91 & 134.06 & 137.28 & 140. & 143.61 & 147.08 & 150.38 & 153.68 & 156.98 & 160.31 & 166 & 173 & 28 & 5 & .50 \\
\hline-0.092 & 132.89 & 133.63 & 135.93 & 139 & 142.32 & 145 & 148. & 1.52 & & 158.76 & & & & & & & \\
\hline-0.094 & 138.05 & 138.79 & 141.09 & 144. & 147. & 150 & & 157 & 160.65 & 163.97 & 167.29 & & 177.31 & & & 19 & 204.02 \\
\hline-0.096 & 143.34 & 144.08 & 146.39 & 149 & 152 & 15 & 159 & 162.67 & 166.00 & 169.32 & 172.67 & 176.01 & & & & & 209.48 \\
\hline-0.098 & 148.78 & 149. & 151.82 & 155 & 158 & 161. & 164 & 168 & & & & & 188.23 & & 66 & 208.38 & 215.10 \\
\hline-0.100 & 154. & 15 & 1 & 160 & 263. & 167. & & & 177 & 180 & 183 & 187.18 & 193 & & & & \\
\hline-0.105 & 168. & 169 & 172 & 175 & 178.48 & 181.80 & 185.32 & 188.49 & 191.84 & 295.1 & 198.57 & 201.95 & 208.72 & & & 22 & \\
\hline-0.110 & 184. & 185.28 & 187. & 190. & 194. & & & 204 & & & 214.34 & 217.74 & 224.56 & 30 & 238.21 & 245.04 & 251.89 \\
\hline-0.215 & & 202.47 & 204.24 & 208.02 & 210 & 214.13 & 217.48 & 220 & 224 & 227 & 231.12 & 54 & 24. & & & & 268.92 \\
\hline-0.120 & 218.90 & 219.65 & 222.01 & 225.22 & 228.57 & 231.91 & 235.33 & 238.72 & 242.14 & 245.50 & & 25 & & & 27 & 28 & \\
\hline-0.125 & 238. & 239.21 & 240 & 244 & 247 & 250 & 254 & 257.76 & 261 & 264.64 & 268.18 & 267.75 & 278 & 285.50 & .48 & .46 & 306.44 \\
\hline-0.130 & 258.01 & 258.76 & 261.12 & 264 & 267. & 271.12 & 274 & 278 & 281 & 284.98 & 288.45 & 292.02 & & & .01 & 320.04 & 327.08 \\
\hline .0 .135 & 280.24 & 281.02 & 282.62 & 286.61 & 289.27 & 292.71 & 296.14 & 299.66 & 303.15 & 306.61 & 312.71 & 313.64 & 320.81 & 327 & 33 & 34 & 348.99 \\
\hline-0.140 & 302.46 & 303.21 & 305.59 & 308.85 & 312.27 & 315.68 & 319.18 & 322.65 & 326.16 & 329.72 & 333.34 & 336.86 & & 351.01 & 35 & 365.26 & 371.39 \\
\hline-0.245 & 327.72 & 328.46 & 330.01 & 334.11 & 336.71 & 340.20 & 343.67 & 347.23 & 350 & 354 & 352.91 & 356.43 & 368.54 & 375.71 & 382.88 & 390.05 & 397.24 \\
\hline-0.150 & 352.97 & 353.71 & 356 & 359.38 & 362.83 & 366.27 & 369.83 & 373. & 376 & 380 & 384.04 & 378.24 & 394.93 & 402.07 & 40 & 41 & 423.75 \\
\hline-0. & 381,64 & 382.39 & 383.84 & 388.07 & 390.59 & 394.16 & 397.62 & 401.22 & 404.80 & 408.76 & 412.09 & 415.57 & 422.96 & 430.08 & 437.35 & 444.62 & 451.91 \\
\hline-0.160 & 410.31 & 411.06 & 413.46 & 426.75 & 420.23 & 423.71 & 427.40 & 430.85 & $431 . .45$ & 438.26 & 441.85 & 445.45 & 452.74 & 459.97 & 467.29 & 474.62 & 482.05 \\
\hline-0.165 & 442.88 & 443.60 & 444.93 & 449.30 & 451.73 & 455.30 & 458.83 & 462.98 & 466.11 & 469.71 & 473.48 & 477.28 & 484.36 & 491.71 & 499.09 & & \\
\hline-0.170 & 475.39 & 476.13 & 478.53 & 81.84 & 35.36 & 88.86 & 92.50 & 96.08 & 99.73 & $503.1,4$ & & & & & & & \\
\hline
\end{tabular}

a See Ref. 1.

ploying parameters from sound velocity measurements by Bartles and Schuele. ${ }^{8}$ The same Murnaghan equation definitely gives too small a compression at high pressures. If one chooses a value of $B_{0}{ }^{\prime}=4.60$ rather than the 5.35 reported by Bartles and Schuele, the resulting Murnaghan equation is in good agreement with the experimental data at low pressures. $B_{0}{ }^{\prime}$ is the pressure derivative of the bulk modulus and is assumed to be independent of pressure in Murnaghan's equation. Even with the smaller choice of $B_{0}{ }^{\prime}$, the Murnaghan equation appears to become definitely less accurate at high pressures.

It is concluded that the value of $B_{0}{ }^{\prime}$ given by Bartles and Schuele is probably too large and that $B_{0}^{\prime}$ for $\mathrm{NaCl}$ must vary with 


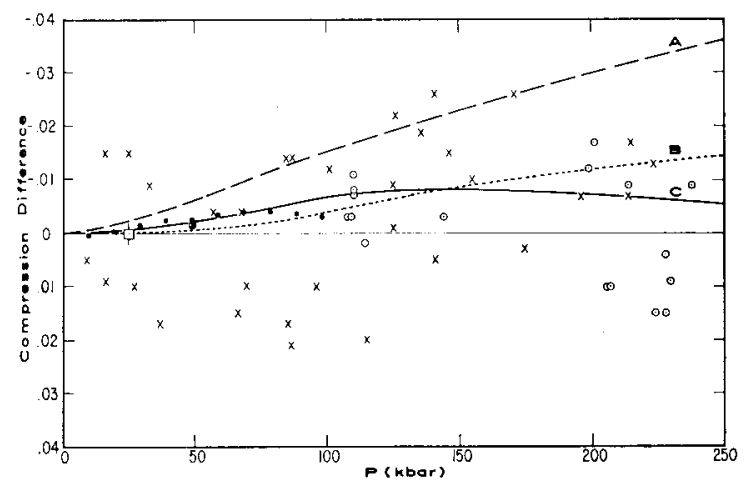

FIG. 1. Comparison of calculated and measured compressions as a function of pressure for $\mathrm{NaCl}$. The ordinate is $\Delta V / V_{0}$ calculated by Decker minu $\Delta V / V_{0}$ calculated or measured by others. Curve A is Murnaghan's equation With $B_{0}$ and $B_{0}$ taken from Ref. 8 . Curve $B$ is Murnaghan's equation with $B_{0}$ as in $A$ but $B_{0}=4,60$. Curve $C$ is calculated by Pere $z$-Aibuerne and ments by ' Ref. 3.

pressure, making the Murnaghan equation a poor equation of state at higher pressures. The experimental data is, however, too scattered to make a choice between the equations of Perez-Albuerne and Drickamer, and the results calculated in Ref. 1 and tabulated in Table I.

I wish to thank Dr. J. Dean Barnett for his help in preparing the table and encouragement in this work.

1 D. L. Decker, J. Appl. Phys. 36, 157 (1965).

2 O. L. Anderson, Proc. Natl. Acad. Sci. U. S. 54, 667 (1965); J. Phys

Chem. Solids 27, $547(1966)$. $H$. G. Drickamer, J. Chem. Phys. 43, 1381 (1965)

4 P. W. Bridgman, Proc. Am. Acad. Arts Sci, 76, 9 (1945).

5 R. H. Christian, Report UCRL-4900, University of California, Lawrence Radiation Laboratory, 1957.

${ }^{6}$ R. N. Jeffrey, J. D. Barnett, H. B. Vanfleet, and H. T. Hall, J. Appl Phys. 37, 3172 (1966).

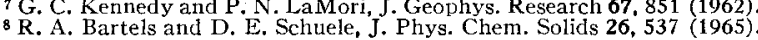

\section{Lorentz Microscopy of Growing Domains in Permalloy Films}

M. D. Coutts AND H. Weinstein

RCA Laboratories, Princelon, N. J.

(Received 8 July 1966)

Q

UANTITATIVE measurements of domain growth in evaporated, strain-sensitive Permalloy $66: 34 \mathrm{Ni}-\mathrm{Fe}$ films of low coercivity have been made using Lorentz electron microscopy. ${ }^{1,2}$ Magnetic ripple ${ }^{1}$ was absent under low axial and radial magnetic fields and present when a small axial field was applied. When magnetic ripple is absent, stages in domain growth can be shown by expressing domain area as a function of the applied field.

For the measurement of domain growth in strain-sensitive materials, it is desirable to have: High resolution by normal electron microscopy and diffraction, an accurate value for the transverse field applied to the specimen, and a low axial field. Previously, measurements have been made in RCA microscopes with an attachment using only a projector lens ${ }^{3,4}$ or in a special specimen holder with axial field coils. ${ }^{5}$ The attachment ${ }^{3,4}$ gives only low magnifications and the film cannot be observed readily by normal microscopy. The specimen holder, while it allows of normal optics, has mainly an axial rather than a transverse field and the transverse component is difficult to measure. A specimen holder was designed which combined the good features of both methods. The specimen holder is shown in Fig. 1. It is made from a standard RCA part; the specimen holder and cap have been machined'to provide space for two miniature coils. Each coil consists of 17 layers of No. 38 Soldereeze wire with 12 turns per layer wound on

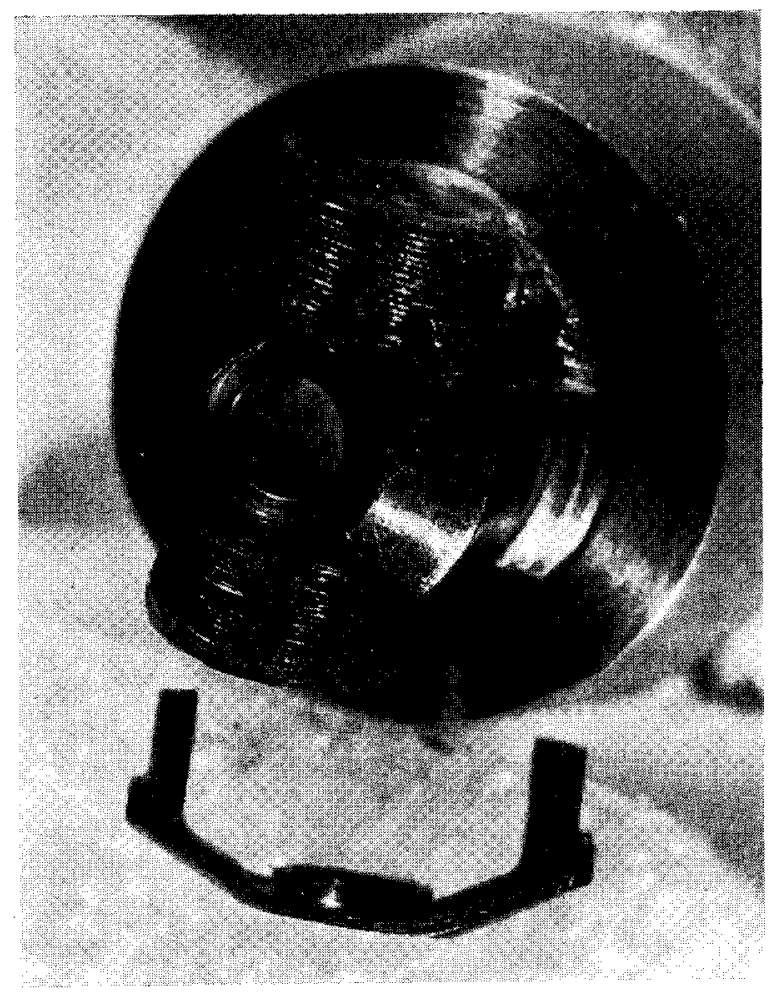

FIG. 1. A modified sample holder for Lorentz microscopy of thin ferromagnetic films in the RCA EMU-3 electron microscope.

a machined Teflon bobbin. The outer radius of each bobbin is $0.098 \mathrm{in}$. and its length $0.028 \mathrm{in}$. The specimen is held at the center of the coils by a small brass cylinder and secured with the cap. The power supply consists of a 12 -V battery, a polarity reversing switch, and voltage divider. ${ }^{5}$ Solenoid current is measured with an ammeter (1.0 A, full scale).

The Helmholtz coils are calibrated using a Hall-effect probe at the specimen level. A maximum field of $120 \mathrm{Oe}$ is obtained for a current of $800 \mathrm{~mA}$. The magnetic field is linear with current and close to the calculated values for the Helmholtz coils.

In operation it is found that the image displacement produced by the miniature Helmholtz coils is easily corrected by a slight lateral movement of the specimen. The advantages of this holder are: Normal operation to $\times 30000$ magnification can be immediately obtained, and selected-area diffraction readily used. Low magnifications of $\times 500$ are obtained by reducing the current in the projector lens. The field applied to the specimen is known with accuracy, the optical system of the microscope is not changed appreciably, and it is possible to change rapidly to high magnifications and selected-area diffraction. Previous sample holders have had the film well above the normal specimen position, and use two or three Helmholt $z$ coils to compensate for deviation of the electron beam.6.7 An axial coil has previously been used in the normal specimen position but has the disadvantage that measurement of absolute values cannot be easily made. ${ }^{5}$ Special Lorentz attachements have also been used but do not provide an immediate change to selected-area diffraction and normal high magnification microscopy ${ }^{3,4}$ since only a projector system is used.

Permalloy films were prepared by evaporation of premelted $66.19 \mathrm{Ni}-33.81 \mathrm{Fe}$ alloy onto a freshly cleaved rocksalt crystal held at $300^{\circ} \mathrm{C}$, in a vacuum of $5 \times 10^{-5}$ Torr. The deposition field was 50 Oe. Film thickness is $800 \AA$ by Tolansky interferometric measurements. Films were floated off, mounted, and examined in the RCA-3G electron microscope at $100 \mathrm{kV}$.

Films were found to have a well-defined uniaxial anisotropy in the direction of the field applied during the evaporation. This ob- 